\title{
On the cardinality and complexity of the set of codings for self-similar sets with positive Lebesgue measure
}

Article

Accepted Version

Baker, S. (2016) On the cardinality and complexity of the set of codings for self-similar sets with positive Lebesgue measure. Monatshefte fur Mathematik, 179 (1). pp. 1-13. ISSN 14365081 doi: https://doi.org/10.1007/s00605-015-0755-2 Available at https://centaur.reading.ac.uk/46864/

It is advisable to refer to the publisher's version if you intend to cite from the work. See Guidance on citing.

To link to this article DOI: http://dx.doi.org/10.1007/s00605-015-0755-2

Publisher: Springer

All outputs in CentAUR are protected by Intellectual Property Rights law, including copyright law. Copyright and IPR is retained by the creators or other copyright holders. Terms and conditions for use of this material are defined in the End User Agreement.

www.reading.ac.uk/centaur 
Central Archive at the University of Reading

Reading's research outputs online 


\title{
ON THE CARDINALITY AND COMPLEXITY OF THE SET OF CODINGS FOR SELF-SIMILAR SETS WITH POSITIVE LEBESGUE MEASURE
}

\author{
SIMON BAKER
}

\begin{abstract}
Let $\lambda_{1}, \ldots, \lambda_{n}$ be real numbers in $(0,1)$ and $p_{1}, \ldots, p_{n}$ be points in $\mathbb{R}^{d}$. Consider the collection of maps $f_{j}: \mathbb{R}^{d} \rightarrow \mathbb{R}^{d}$ given by

$$
f_{j}(x)=\lambda_{j} x+\left(1-\lambda_{j}\right) p_{j} .
$$

It is a well known result that there exists a unique nonempty compact set $\Lambda \subset \mathbb{R}^{d}$ satisfying $\Lambda=\cup_{j=1}^{n} f_{j}(\Lambda)$. Each $x \in \Lambda$ has at least one coding, that is a sequence $\left(\epsilon_{i}\right)_{i=1}^{\infty} \in\{1, \ldots, n\}^{\mathbb{N}}$ that satisfies $\lim _{N \rightarrow \infty} f_{\epsilon_{1}} \cdots f_{\epsilon_{N}}(0)=x$.

We study the size and complexity of the set of codings of a generic $x \in \Lambda$ when $\Lambda$ has positive Lebesgue measure. In particular, we show that under certain natural conditions almost every $x \in \Lambda$ has a continuum of codings. We also show that almost every $x \in \Lambda$ has a universal coding.

Our work makes no assumptions on the existence of holes in $\Lambda$ and improves upon existing results when it is assumed $\Lambda$ contains no holes.
\end{abstract}

\section{INTRODUCTION}

Let $\lambda \in\left(\frac{1}{2}, 1\right)$ and $I_{\lambda}:=\left[0, \frac{\lambda}{1-\lambda}\right]$. Each $x \in I_{\lambda}$ admits a sequence $\left(\epsilon_{i}\right)_{i=1}^{\infty} \in\{0,1\}^{\mathbb{N}}$ such that

$$
x=\sum_{i=1}^{\infty} \epsilon_{i} \lambda^{i} .
$$

Such a sequence is called a $\lambda$-expansion for $x$. Expansions of this form were pioneered in the papers of Rényi [21] and Parry [18]. We can study $\lambda$-expansions via the iterated function system defined by the maps $f_{0}(x)=\lambda x$ and $f_{1}(x)=\lambda x+\lambda . I_{\lambda}$ is the attractor of the IFS $\left\{f_{0}, f_{1}\right\}$ and it is a straightforward exercise to show that

$$
f_{\epsilon_{1}} \cdots f_{\epsilon_{N}}(0)=\sum_{i=1}^{N} \epsilon_{i} \lambda^{i} .
$$

Therefore $\lim _{N \rightarrow \infty} f_{\epsilon_{1}} \cdots f_{\epsilon_{N}}(0)=x$ if and only if $\left(\epsilon_{i}\right)_{i=1}^{\infty}$ is a $\lambda$-expansion for $x$.

In [6] it was shown that if $\lambda \in\left(\frac{\sqrt{5}-1}{2}, 1\right)$ then every $x \in\left(0, \frac{\lambda}{1-\lambda}\right)$ has a continuum of $\lambda$ expansions. The endpoints of $I_{\lambda}$ trivially have a unique expansion. In [5] the value $\frac{\sqrt{5}-1}{2}$ was shown to be sharp in the following sense: If $\lambda \in\left(\frac{1}{2}, \frac{\sqrt{5}-1}{2}\right)$ then there exists $x \in\left(0, \frac{\lambda}{1-\lambda}\right)$ with a unique $\lambda$-expansion. The size of the set of points with unique $\lambda$-expansion was studied further in [11], amongst other things it was shown that the set of $x \in I_{\lambda}$ with unique $\lambda$-expansion

Date: February 4, 2015.

2010 Mathematics Subject Classification. 28A80, 37A45.

Key words and phrases. Iterated function systems, Beta-expansions. 
has positive Hausdorff dimension when $\lambda \in\left(\frac{1}{2}, \lambda^{*}\right)$. Here $\lambda^{*} \approx 0.559$ is the reciprocal of the Komornik Loreti constant introduced in [17]. However, in [22] it was shown that Lebesgue almost every $x \in I_{\lambda}$ has a continuum of $\lambda$-expansions for any $\lambda \in\left(\frac{1}{2}, 1\right)$. This almost every result was later generalised to a class of IFS's in [23]. We now give details of their generalisation.

Let $\lambda_{1}, \ldots, \lambda_{n}$ be real numbers in $(0,1)$ and $p_{1}, \ldots, p_{n}$ be points in $\mathbb{R}^{d}$. Consider the collection of maps $f_{j}: \mathbb{R}^{d} \rightarrow \mathbb{R}^{d}$ given by

$$
f_{j}(x)=\lambda_{j} x+\left(1-\lambda_{j}\right) p_{j}
$$

In [12] it was shown that there exists a unique nonempty compact set $\Lambda \subset \mathbb{R}^{d}$ that satisfies $\Lambda=\cup_{j=1}^{n} f_{j}(\Lambda)$. We refer to $\Lambda$ as the attractor for the collection of maps $\left\{f_{j}\right\}_{j=1}^{n}$, or when the collection of maps is obvious just the attractor. Each $x \in \Lambda$ admits a sequence $\left(\epsilon_{i}\right)_{i=1}^{\infty} \in$ $\{1, \ldots, n\}^{\mathbb{N}}$ such that $\lim _{N \rightarrow \infty} f_{\epsilon_{1}} \cdots f_{\epsilon_{N}}(0)=x$. We refer to such a sequence as a coding for $x$. Moreover, the set of $x$ which have a coding is precisely $\Lambda$. When $\lambda_{1}=\cdots=\lambda_{n}$ we will say that we are in the homogeneous case. When there exists $\lambda_{i}, \lambda_{j}$ such that $\lambda_{i} \neq \lambda_{j}$ we will say that we are in the inhomogeneous case. When we are in the homogeneous case we will denote the common scaling ratio by $\lambda$. For further background we refer the reader to Chapter 1 from [15] and Chapter 9 from [8].

Let $\Omega$ denote the convex hull of $\left\{p_{1}, \ldots, p_{n}\right\}$. Without loss of generality we may assume that the dimension of $\Omega$ is $d$. In [23] the author considers the homogeneous case under the additional assumption that $\Lambda=\Omega$, i.e., the case when the attractor has no holes. In particular it is shown that the property $\Lambda=\Omega$ holds for all $\lambda \geq \frac{d}{d+1}$. He also proved the following result.

Theorem 1.1. Assume $\Lambda=\Omega$ and that we are in the homogeneous case. If there exists $1 \leq k<$ $l \leq n$ such that a vertex of $f_{k}(\Omega)$ belongs to the interior of $f_{l}(\Omega)$ then Lebesgue almost every $x \in \Lambda$ has a continuum of codings, and the exceptional set has Hausdorff dimension strictly less than $d$.

The purpose of this paper is to generalise and strengthen Theorem 1.1. Our approach does not make any assumptions on the existence of holes in $\Lambda$ and extends to the inhomogeneous case.

Let $\Lambda$ be as above. We will be interested in the case when $\mathcal{L}(\Lambda)>0$. Here $\mathcal{L}(\cdot)$ denotes the $d$-dimensional Lebesgue measure. Clearly when $\Lambda=\Omega$ then $\mathcal{L}(\Lambda)>0$. However, there are cases when $\Lambda \neq \Omega$, i.e., the case when our attractor has holes, yet the Lebesgue measure of $\Lambda$ is still positive. Typically, determining whether the attractor of a given IFS has positive Lebesgue measure is a difficult problem. For more on this difficult problem we refer the reader to [9] and [25].

In [13] the authors consider the case when there are $n$ homogeneous contractions $f_{j}: \mathbb{R}^{2} \rightarrow \mathbb{R}^{2}$ of the form

$$
f_{j}(x)=\lambda x+\left(c_{j}^{1}, c_{j}^{2}\right)
$$

where $\left(c_{j}^{1}, c_{j}^{2}\right) \in\left\{(a, b) \in \mathbb{Z}^{2}: 0 \leq a, b \leq k-1\right\}$. It is assumed that $n>k$. If the points $\left(c_{j}^{1}, c_{j}^{2}\right)$ are fixed and $\lambda$ is allowed to vary, the geometry of the associated $\Lambda$ also varies. In particular, if $\lambda$ is sufficiently small then the open set condition is satisfied and the Hausdorff dimension is easy to compute. However, for $\lambda$ sufficiently large the open set condition is not satisfied and determining the dimension of $\Lambda$ is less straightforward. The authors show that for each family of contractions there exists an interval $I \subset(0,1)$ for which $\mathcal{L}(\Lambda)>0$ for almost every $\lambda \in I$. 
Moreover, this $I$ is calculated explicitly. Their results imply the existence of a broad class of $\Lambda$ for which $\mathcal{L}(\Lambda)>0$ and $\Lambda$ contains holes.

In [2] the case where $p_{1}, p_{2}$ and $p_{3}$ are the vertices of an equilateral triangle is studied. For $\lambda=\frac{1}{2}$ our $\Lambda$ is the well known Sierpiński gasket. It can be shown that $\Lambda=\Omega$ if and only if $\lambda \geq \frac{2}{3}$. The authors show that for all $\lambda \geq \lambda^{*} \approx 0.647$ the associated $\Lambda$ has nonempty interior and therefore has positive Lebesgue measure. Here $\lambda^{*}$ is the appropriate root of $2 x^{3}-2 x^{2}+2 x=1$. It is a consequence of the aforementioned results of [13] that for almost every $\lambda>0.585 \ldots$ the associated $\Lambda$ has positive Lebesgue measure.

Our main result is the following generalisation of Theorem 1.1.

Theorem 1.2. Assume $\sum_{j=1}^{n} \lambda_{j}^{d} \neq 1$ and that $\mathcal{L}(\Lambda)>0$. Then almost every $x \in \Lambda$ has $a$ continuum of codings.

When $f_{k}(\Lambda) \cap f_{l}(\Lambda)$ has nonempty interior for some $1 \leq k<l \leq n$ we can make a stronger statement.

Theorem 1.3. Assume $f_{k}(\Lambda) \cap f_{l}(\Lambda)$ has nonempty interior for some $1 \leq k<l \leq n$. Then the set of points that do not have a continuum of codings has Hausdorff dimension strictly less that $d$.

The expression $\sum_{j=1}^{n} \lambda_{j}^{d}$ occuring in the statement of Theorem 1.2 appears naturally in the study of IFS's. If $\sum_{j=1}^{n} \lambda_{j}^{d}<1$ then it is a simple exercise to show $\mathcal{L}(\Lambda)=0$. Therefore it is only possible for $\mathcal{L}(\Lambda)>0$ when $\sum_{j=1}^{n} \lambda_{j}^{d} \geq 1$. The condition $\sum_{j=1}^{n} \lambda_{j}^{d} \neq 1$ stated in Theorem 1.2 is not a technical condition and is in fact essential. It will be shown in Corollary 2.4 that if $\sum_{j=1}^{n} \lambda_{j}^{d}=1$ then almost every $x \in \Lambda$ has a unique coding. It is natural to ask whether there exists self-similar sets with positive Lebesgue measure when $\sum_{j=1}^{n} \lambda_{j}^{d}=1$. However, it is straightforward to construct examples when this equation is satisfied. For example, consider the case where $d=1, p_{1}=0, p_{2}=1$ and $\lambda_{1}=\lambda_{2}$. In this case $\sum_{j=1}^{n} \lambda_{j}=1$ when $\lambda=\frac{1}{2}$. The associated $\Lambda$ is the interval $[0,1]$, which clearly has positive Lebesgue measure.

In this paper, as well as studying the cardinality of the set of codings of a generic $x \in \Lambda$, we also study the complexity of these codings. In the context of $\lambda$-expansions we say that a $\lambda$-expansion of $x$, the sequence $\left(\epsilon_{i}\right)_{i=1}^{\infty} \in\{0,1\}^{\mathbb{N}}$, is a universal expansion for $x$ if given any finite block $\delta_{1} \cdots \delta_{N}$ consisting of 0 's and 1's, there exists $k \in \mathbb{N}$ such that $\epsilon_{k+i}=\delta_{i}$ for $1 \leq i \leq N$. Universal expansions were originally introduced in [7], where they were shown to be intimately related to the so called spectra of a real number. We discuss this relation in more detail in Section 5. In [24] it was shown that for $\lambda \in\left(\frac{1}{2}, 1\right)$ almost every $x \in I_{\lambda}$ has a universal expansion, this was later improved upon in [3] where it was shown that almost every $x \in I_{\lambda}$ has $2^{\aleph_{0}}$ universal expansions. Proceeding by analogy with the case of $\lambda$-expansions, given $x \in \Lambda$ and $\left(\epsilon_{i}\right)_{i=1}^{\infty} \in\{1, \ldots, n\}^{\mathbb{N}}$ a coding for $x$. We say that $\left(\epsilon_{i}\right)_{i=1}^{\infty}$ is a universal coding if for any finite block $\delta_{1} \cdots \delta_{N}$ consisting of elements from $\{1, \ldots, n\}$, there exists $k \in \mathbb{N}$ such that $\epsilon_{k+i}=\delta_{i}$ for $1 \leq i \leq N$. Our result regarding universal codings is the following.

Theorem 1.4. Suppose $\mathcal{L}(\Lambda)>0$, then almost every $x \in \Lambda$ has a universal coding. 
The proofs of Theorems 1.2 and 1.4 will take on a similar structure. As such we will only prove Theorem 1.2 in full and outline the necessary modifications required to prove Theorem 1.4 .

The rest of this paper is structured as follows. In Section 2 we state some necessary preliminaries before giving our proofs of Theorems 1.2, 1.3 and 1.4 in Section 3. In Section 4 we discuss some applications of our results to $\lambda$-expansions with arbitrary digit sets. Finally in Section 5 we pose some open questions.

\section{Preliminaries}

Before proving Theorems 1.2, 1.3 and 1.4 we require the following technical arguments. For ease of exposition we denote the set of codings for a given $x \in \Lambda$ by $\Sigma_{\Lambda}(x)$, i.e.,

$$
\Sigma_{\Lambda}(x):=\left\{\left(\epsilon_{i}\right)_{i=1}^{\infty} \in\{1, \ldots, n\}^{\mathbb{N}}: \lim _{N \rightarrow \infty} f_{\epsilon_{1}} \cdots f_{\epsilon_{N}}(0)=x\right\} .
$$

Moreover, let

$$
U_{\Lambda}:=\left\{x \in \Lambda: \operatorname{card} \Sigma_{\Lambda}(x)=1\right\}
$$

That is $U_{\Lambda}$ is the set of points with a unique coding. $U_{\Lambda}$ is called the set of uniqueness. Understanding the size/dimension of this set will be important in our proofs of Theorems 1.2 and 1.3 .

Let $\left\{B_{k}\right\}_{k=1}^{\infty}$ be an enumeration of the set of all finite blocks consisting of elements from the set $\{1, \ldots, n\}$. Moreover let $N_{k}$ denote the length of the block $B_{k}$. To each $B_{k}$ we associate the set $U_{B_{k}}$ defined as follows:

$$
U_{B_{k}}:=\left\{x \in \Lambda: \text { no coding of } x \text { contains the block } B_{k}\right\} .
$$

The following proposition highlights the importance of the set $U_{\Lambda}$ and the $U_{B_{k}}$ 's.

Proposition 2.1. The following inclusions hold:

$$
\begin{gathered}
U_{\Lambda} \subseteq\left\{x \in \Lambda: \text { card } \Sigma_{\Lambda}(x)<2^{\aleph_{0}}\right\} \\
\bigcup_{k=1}^{\infty} U_{B_{k}} \subseteq\{x \in \Lambda: x \text { has no universal coding }\} \\
\left\{x \in \Lambda: \text { card } \Sigma_{\Lambda}(x)<2^{\aleph_{0}}\right\} \subseteq \bigcup_{N=0}^{\infty} \bigcup_{\left(\epsilon_{i}\right) \in\{1, \ldots, n\}^{N}} f_{\epsilon_{1}} \cdots f_{\epsilon_{N}}\left(U_{\Lambda}\right), \\
\{x \in \Lambda: x \text { has no universal coding }\} \subseteq \bigcup_{k=1}^{\infty} \bigcup_{N=0}^{\infty} \bigcup_{\left(\epsilon_{i}\right) \in\{1, \ldots, n\}^{N}} f_{\epsilon_{1}} \cdots f_{\epsilon_{N}}\left(U_{B_{k}}\right) .
\end{gathered}
$$


Proof. Statements (2.1) and (2.2) are obvious. The proof of (2.3) is a simple generalisation of the proof of Lemma 3.1 from [23]. As such we only show that (2.4) holds. Suppose $x \in \Lambda$ does not have a universal coding and let $\left\{B_{k}\right\}_{k=1}^{\infty}$ be as above. To begin with we ask whether $x$ has a coding containing the block $B_{1}$. If it doesn't then $x \in U_{B_{1}}$. Suppose otherwise and let $\left(\epsilon_{i}\right)_{i=1}^{\infty} \in$ $\Sigma_{\Lambda}(x)$ contain $B_{1}$. Let $M_{1} \in \mathbb{N}$ be such that $\epsilon_{M_{1}+1} \cdots \epsilon_{M_{1}+N_{1}}=B_{1}$. Moreover, let $j_{1} \in \mathbb{N}$ be the unique natural number for which $B_{k}$ appears in $\epsilon_{1} \cdots \epsilon_{M_{1}+N_{1}}$ for every $1 \leq k \leq j_{1}$, but $B_{j_{1}+1}$ does not appear in $\epsilon_{1} \cdots \epsilon_{M_{1}+N_{1}}$. Such a $j_{1}$ has to exist as $\epsilon_{1} \cdots \epsilon_{M_{1}+N_{1}}$ is of finite length. Now we consider all codings of $x$ that begin with $\epsilon_{1} \cdots \epsilon_{M_{1}+N_{1}}$ and ask whether one of these codings contains the block $B_{j_{1}+1}$. If there doesn't exist such a coding then $\lim _{N \rightarrow \infty} f_{\epsilon_{M_{1}+N_{1}+1}} \cdots f_{\epsilon_{N}}(0) \in$ $U_{B_{j_{1}+1}}$, which implies $x \in f_{1} \cdots f_{\epsilon_{M_{1}+N_{1}}}\left(U_{B_{j_{1}}+1}\right)$. If there does exist such a coding we denote it by $\left(\epsilon_{i}^{2}\right)_{i=1}^{\infty}$ and let $M_{2} \in \mathbb{N}$ be such that $\epsilon_{M_{2}+1}^{2} \cdots \epsilon_{M_{2}+N_{j_{1}+1}}^{2}=B_{j_{1}+1}$. We then define $j_{2} \in \mathbb{N}$ to be the unique natural number such that $B_{k}$ appears in $\epsilon_{1}^{2} \cdots \epsilon_{M_{2}+N_{j_{1}+1}}^{2}$ for all $1 \leq k \leq j_{2}$, but $B_{j_{2}+1}$ does not appear. We then ask whether there exists a coding for $x$ beginning with $\epsilon_{1}^{2} \cdots \epsilon_{M_{2}+N_{j_{1}+1}}^{2}$ that contains the block $B_{j_{2}+1}$. If such a coding doesn't exist we stop, if one does exist we repeat the above steps. Assuming the above process does not terminate then at the $n$-th iteration we have constructed a finite sequence containing the blocks $B_{1}, \ldots, B_{n}$, and this sequence can be extended to an element of $\Sigma_{\Lambda}(x)$. If this process continues indefinitely then we will construct a universal coding for $x$. However, as $x$ has no universal coding this algorithm must at some point terminate. This yields $K, M_{K} \in \mathbb{N}$ and $\left(\epsilon_{i}\right)_{i=1}^{\infty} \in \Sigma_{\Lambda}(x)$ such that $\lim _{N \rightarrow \infty} f_{\epsilon_{M_{K}+1}} \cdots f_{\epsilon_{N}}(0) \in U_{B_{K}}$. In which case $x \in f_{\epsilon_{1}} \cdots f_{\epsilon_{M_{K}}}\left(U_{B_{K}}\right)$ and we may deduce the inclusion in (2.4).

The right hand side of (2.4) in Proposition 2.1 might seem excessive. We might naively expect that if $x \in \Lambda$ does not have a universal coding then $x \in U_{B_{k}}$, for some $k$. However, even if $x$ has no universal coding we cannot discount the possibility that for each $B_{k}$ there exists $\left(\epsilon_{i}^{k}\right)_{i=1}^{\infty} \in \Sigma_{\Lambda}(x)$ containing $B_{k}$.

The following corollary is an immediate consequence of Proposition 2.1 and the fact that our $f_{j}$ 's are all similitudes.

Corollary 2.2. The following statements hold:

- $\mathcal{L}\left(\left\{x \in \Lambda:\right.\right.$ card $\left.\left.\Sigma_{\Lambda}(x)<2^{\aleph_{0}}\right\}\right)=0$ if and only if $\mathcal{L}\left(U_{\Lambda}\right)=0$.

- $\operatorname{dim}_{H}\left(\left\{x \in \Lambda: \operatorname{card} \Sigma_{\Lambda}(x)<2^{\aleph_{0}}\right\}\right)=\operatorname{dim}_{H}\left(U_{\Lambda}\right)$.

- $\mathcal{L}(\{x \in \Lambda: x$ has no universal coding $\})=0$ if and only if $\mathcal{L}\left(U_{B_{k}}\right)=0$ for every $B_{k}$.

By Corollary 2.2, to show that Theorems 1.2, 1.3 and 1.4 hold, it suffices to show that equivalent statements hold for $U_{\Lambda}$ and a typical $U_{B_{k}}$.

We now elaborate on the technical condition $\sum_{j=1}^{n} \lambda_{j}^{d} \neq 1$ stated in Theorem 1.2.

Proposition 2.3. Assume $\mathcal{L}(\Lambda)>0$. If $\sum_{j=1}^{n} \lambda_{j}^{d}=1$ then $\mathcal{L}\left(f_{k}(\Lambda) \cap f_{l}(\Lambda)\right)=0$ for all $1 \leq k<$ $l \leq n$. However, if $\sum_{j=1}^{n} \lambda_{j}^{d} \neq 1$ there exists $1 \leq k<l \leq n$ such that $\mathcal{L}\left(f_{k}(\Lambda) \cap f_{l}(\Lambda)\right)>0$. 
Proof. It is a straighforward inductive argument to show that the following holds. Let $\left\{A_{j}\right\}_{j=1}^{n}$ be a finite collection of measurable sets with finite Lebesgue measure. Then

$$
\mathcal{L}\left(\cup_{j=1}^{n} A_{j}\right)=\sum_{j=1}^{n} \mathcal{L}\left(A_{j}\right)-\sum_{i=2}^{n} \mathcal{L}\left(\cup_{j=1}^{i-1} A_{j} \cap A_{i}\right) .
$$

Let us assume $\sum_{j=1}^{n} \lambda_{j}^{d}=1$ and that there exists $1 \leq k<l \leq n$ such that $\mathcal{L}\left(f_{k}(\Lambda) \cap f_{l}(\Lambda)\right)>0$. Without loss of generality we may assume that $k=1$ and $l=2$. We observe the following:

$$
\begin{aligned}
\mathcal{L}(\Lambda) & =\mathcal{L}\left(\cup_{j=1}^{n} f_{j}(\Lambda)\right) \\
& =\sum_{j=1}^{n} \mathcal{L}\left(f_{j}(\Lambda)\right)-\sum_{i=2}^{n} \mathcal{L}\left(\cup_{j=1}^{i-1} f_{j}(\Lambda) \cap f_{i}(\Lambda)\right) \\
& =\mathcal{L}(\Lambda) \sum_{j=1}^{n} \lambda_{j}^{d}-\sum_{i=2}^{n} \mathcal{L}\left(\cup_{j=1}^{i-1} f_{j}(\Lambda) \cap f_{i}(\Lambda)\right) .
\end{aligned}
$$

In our second equality we have used equation (2.5). It follows that

$$
0=\sum_{i=2}^{n} \mathcal{L}\left(\cup_{j=1}^{i-1} f_{j}(\Lambda) \cap f_{i}(\Lambda)\right) .
$$

However, this is not possible if $\mathcal{L}\left(f_{1}(\Lambda) \cap f_{2}(\Lambda)\right)>0$.

Now let us assume that $\sum_{j=1}^{n} \lambda_{j}^{d} \neq 1$ and that $\mathcal{L}(\Lambda)>0$. By the inclusion exclusion principle the following equation holds.

$$
\begin{aligned}
\mathcal{L}(\Lambda) & =\mathcal{L}\left(\cup_{j=1}^{n} f_{j}(\Lambda)\right) \\
& =\sum_{j=1}^{n} \mathcal{L}\left(f_{j}(\Lambda)\right)-\sum_{1 \leq i<j \leq n}^{n} \mathcal{L}\left(f_{i}(\Lambda) \cap f_{j}(\Lambda)\right)+\sum_{1 \leq i<j<h \leq n} \mathcal{L}\left(f_{i}(\Lambda) \cap f_{j}(\Lambda) \cap f_{h}(\Lambda)\right)- \\
& \cdots+(-1)^{n-1} \mathcal{L}\left(\cap_{j=1}^{n} f_{j}(\Lambda)\right) .
\end{aligned}
$$

Which by a simple manipulation implies

$$
\begin{gathered}
\left(\sum_{j=1}^{n} \lambda_{j}^{d}-1\right) \mathcal{L}(\Lambda)=\sum_{1 \leq i<j \leq n}^{n} \mathcal{L}\left(f_{i}(\Lambda) \cap f_{j}(\Lambda)\right)-\sum_{1 \leq i<j<h \leq n} \mathcal{L}\left(f_{i}(\Lambda) \cap f_{j}(\Lambda) \cap f_{k}(\Lambda)\right)+ \\
\cdots+(-1)^{n} \mathcal{L}\left(\cap_{j=1}^{n} f_{j}(\Lambda)\right) .
\end{gathered}
$$

By our assumptions the left hand side of the above equation is not equal to zero. This implies the right hand side is also non zero and there must exist $1 \leq k<l \leq n$ such that $\mathcal{L}\left(f_{k}(\Lambda) \cap f_{l}(\Lambda)\right)>$ 0 .

We remark that if $x \in f_{k}(\Lambda) \cap f_{l}(\Lambda)$ with $k \neq l$ then $x$ has at least two codings, one with first digit $k$ and one with first digit $l$. Moreover, it is straightforward to show that $x \in$ $f_{\epsilon_{1}} \cdots f_{\epsilon_{N}}\left(f_{k}(\Lambda) \cap f_{l}(\Lambda)\right)$ for some $N \in \mathbb{N},\left(\epsilon_{i}\right)_{i=1}^{N} \in\{1, \ldots, n\}^{N}$ and $1 \leq k<l \leq n$ if and only if $x$ has at least two codings. This important remark will be used in the proof of the following corollary and later in our proof of Theorem 1.2. 
Corollary 2.4. Suppose $\sum_{j=1}^{n} \lambda_{j}^{d}=1$ and $\mathcal{L}(\Lambda)>0$. Then almost every $x \in \Lambda$ has a unique coding.

Proof. By the above remarks the following equality holds

$$
\left\{x \in \Lambda: \operatorname{card} \Sigma_{\Lambda}(x)>1\right\}=\bigcup_{1 \leq k<l \leq n} \bigcup_{N=0}^{\infty} \bigcup_{\left(\epsilon_{i}\right) \in\{1, \ldots, n\}^{N}} f_{\epsilon_{1}} \cdots f_{\epsilon_{N}}\left(f_{k}(\Lambda) \cap f_{l}(\Lambda)\right)
$$

It is an immediate consequence of this equality, the fact that our $f_{j}$ 's are all similitudes, and Proposition 2.3 that $\mathcal{L}\left(\left\{x \in \Lambda: \operatorname{card} \Sigma_{\Lambda}(x)>1\right\}\right)=0$.

\section{Proof of TheOrems $1.2,1.3$ AND 1.4}

We begin by proving Theorems 1.2 and 1.4. Their proofs will depend on an application of the Lebesgue density theorem. The Lebesgue density theorem states that if $E \subset \mathbb{R}^{d}$ is a Lebesgue measurable set, then for almost every $x \in E$

$$
\lim _{r \rightarrow 0} \frac{\mathcal{L}\left(E \cap B_{r}(x)\right)}{\mathcal{L}\left(B_{r}(x)\right)}=1
$$

Here $B_{r}(x)$ denotes the closed $d$-dimensional ball in $\mathbb{R}^{d}$ with radius $r$ centred at $x$. This statement is of course vacuous if $\mathcal{L}(E)=0$. It is an immediate consequence of the Lebesgue density theorem that if $E \subset \mathbb{R}^{d}$ is such that every $x \in E$ satisfies

$$
\limsup _{r \rightarrow 0} \frac{\mathcal{L}\left(E \cap B_{r}(x)\right)}{\mathcal{L}\left(B_{r}(x)\right)}<1
$$

then $\mathcal{L}(E)=0$. This will be the strategy we employ when it comes to proving Theorems 1.2 and 1.4 .

Proof of Theorem 1.2. By Corollary 2.2 it suffices to show $\mathcal{L}\left(U_{\Lambda}\right)=0$. By Proposition 2.3 we may assume that $1 \leq k<l \leq n$ are such that $\mathcal{L}\left(f_{k}(\Lambda) \cap f_{l}(\Lambda)\right)>0$.

We now fix $x \in U_{\Lambda}$, and let $\left(\epsilon_{i}\right)_{i=1}^{\infty}$ be its unique coding. Given $r>0$ we associate the unique $n(r) \in \mathbb{N}$ satisfying

$$
\operatorname{Diam}(\Omega) \prod_{i=1}^{n(r)} \lambda_{\epsilon_{i}}<r \leq \operatorname{Diam}(\Omega) \prod_{i=1}^{n(r)-1} \lambda_{\epsilon_{i}}
$$

It is a consequence of these inequalities that $f_{\epsilon_{1}} \cdots f_{\epsilon_{n(r)}}(\Lambda) \subset B_{r}(x)$. 
We observe the following:

$$
\begin{aligned}
\frac{\mathcal{L}\left(U_{\Lambda} \cap B_{r}(x)\right)}{\mathcal{L}\left(B_{r}(x)\right)} & =1-\frac{\mathcal{L}\left(U_{\Lambda}^{c} \cap B_{r}(x)\right)}{\mathcal{L}\left(B_{r}(x)\right)} \\
& \leq 1-\frac{\mathcal{L}\left(U_{\Lambda}^{c} \cap f_{\epsilon_{1}} \cdots f_{\epsilon_{n(r)}}(\Lambda)\right)}{\mathcal{L}\left(B_{r}(x)\right)} \\
& \leq 1-\frac{\mathcal{L}\left(f_{\epsilon_{1}} \cdots f_{\epsilon_{n(r)}}\left(f_{k}(\Lambda) \cap f_{l}(\Lambda)\right)\right)}{\mathcal{L}\left(B_{r}(x)\right)} \\
& =1-\frac{\mathcal{L}\left(f_{k}(\Lambda) \cap f_{l}(\Lambda)\right) \prod_{i=1}^{n(r)} \lambda_{\epsilon_{i}}^{d}}{C(d) r^{d}} \\
& \leq 1-\frac{\mathcal{L}\left(f_{k}(\Lambda) \cap f_{l}(\Lambda)\right) \prod_{i=1}^{n(r)} \lambda_{\epsilon_{i}}^{d}}{C(d)\left(\operatorname{Diam}(\Omega) \prod_{i=1}^{n(r)-1} \lambda_{\epsilon_{i}}\right)^{d}} \\
& =1-\frac{\mathcal{L}\left(f_{k}(\Lambda) \cap f_{l}(\Lambda)\right) \lambda_{n(r)}^{d}}{C(d) D i a m(\Omega)^{d}} \\
& =1-\frac{\mathcal{L}\left(f_{k}(\Lambda) \cap f_{l}(\Lambda)\right) \min _{1 \leq j \leq n}\left\{\lambda_{j}^{d}\right\}}{C(d) \operatorname{Diam}(\Omega)^{d}} .
\end{aligned}
$$

In the third line of the above we have used the fact that $f_{\epsilon_{1}} \cdots f_{\epsilon_{n(r)}}\left(f_{k}(\Lambda) \cap f_{l}(\Lambda)\right) \subset f_{1} \cdots f_{\epsilon_{n(r)}}(\Lambda)$ and $f_{\epsilon_{1}} \cdots f_{\epsilon_{n(r)}}\left(f_{k}(\Lambda) \cap f_{l}(\Lambda)\right) \subset U_{\Lambda}^{c}$. Here $C(d)$ is the $d$-dimensional volume of the unit sphere. Clearly the upper density can therefore always be bounded above by some positive constant strictly less than 1 . Which by our earlier remarks implies $\mathcal{L}\left(U_{\Lambda}\right)=0$.

By Corollary 2.2 to prove Theorem 1.4 it suffices to show $\mathcal{L}\left(U_{B_{k}}\right)=0$ for each $B_{k}$. This will follow from an analogous application of the Lebesgue density theorem. The role of $f_{k}(\Lambda) \cap f_{l}(\Lambda)$ is played by $f_{\epsilon_{1}} \cdots f_{\epsilon_{N_{k}}}(\Lambda)$ where $B_{k}=\epsilon_{1} \cdots \epsilon_{N_{k}}$. Clearly $f_{\epsilon_{1}} \cdots f_{\epsilon_{N_{k}}}(\Lambda) \not \subset U_{B_{k}}$, it has measure $\mathcal{L}(\Lambda) \prod_{i=1}^{N_{k}} \lambda_{\epsilon_{i}}^{d}$ and its image under any finite sequence of $f_{j}$ 's will also be in the complement of $U_{B_{k}}$.

We now prove Theorem 1.3. The proof of this theorem is analogous to the proof of Theorem 1.1 with one minor alteration. We begin by stating a lemma whose proof can be found in [23].

Lemma 3.1. Let $A \subset \mathbb{R}^{d}$ be such that there exists a positive constant $\delta>0$ such that for an arbitrary cube $C \subset \mathbb{R}^{d}$ which intersects $A$, one can find a cube $C_{0} \subset C$ such that $\mathcal{L}\left(C_{0}\right) \geq$ $\delta \mathcal{L}(C)$ and $C_{0} \cap A=\emptyset$. Then $\operatorname{dim}_{H}(A)<d$.

The proof of Lemma 3.1 is fairly straighforward and follows from a box counting argument.

Proof of Theorem 1.3. By Corollary 2.2 it suffices to show $\operatorname{dim}_{H}\left(U_{\Lambda}\right)<d$. We now show that Lemma 3.1 can be applied with $A=U_{\Lambda}$. By our assumption $f_{k}(\Lambda) \cap f_{l}(\Lambda)$ has nonempty interior and therefore contains a $d$-dimensional cube that we shall denote by $C^{*}$. We will show that we can take

$$
\delta=\min \left\{2^{-d}, \frac{\mathcal{L}\left(C^{*}\right) \min _{1 \leq j \leq n}\left\{\lambda_{j}^{d}\right\}}{(2 \operatorname{Diam}(\Omega))^{d}}\right\}
$$


Let $C(z, r)$ denote the cube in $\mathbb{R}^{d}$ centred at $z$ with side length $r$. Suppose $C(z, r)$ intersects $U_{\Lambda}$. We ask whether $U_{\Lambda}$ intersects $C\left(z, \frac{r}{2}\right)$. If it doesn't we can take $C_{0}=C\left(z, \frac{r}{2}\right)$ and $\mathcal{L}\left(C_{0}\right)=$ $2^{-d} \mathcal{L}(C(z, r))$. Suppose otherwise, let $x \in U_{\Lambda} \cap C\left(z, \frac{r}{2}\right)$ and $\left(\epsilon_{i}\right)_{i=1}^{\infty} \in \Sigma_{\Lambda}(x)$. We let $n(r) \in \mathbb{N}$ denote the unique natural number satisfying the following inequalities

$$
\operatorname{Diam}(\Omega) \prod_{i=1}^{n(r)} \lambda_{\epsilon_{i}}<\frac{r}{2} \leq \operatorname{Diam}(\Omega) \prod_{i=1}^{n(r)-1} \lambda_{\epsilon_{i}}
$$

Clearly $f_{\epsilon_{1}} \cdots f_{\epsilon_{n(r)}}(\Lambda) \subset C(z, r)$ and therefore $f_{\epsilon_{1}} \cdots f_{\epsilon_{n(r)}}\left(C^{*}\right) \subset C(z, r)$. Moreover $f_{\epsilon_{1}} \cdots f_{\epsilon_{n(r)}}\left(C^{*}\right)$ is a cube and it is contained in $U_{\Lambda}^{c}$. Finally we observe

$$
\mathcal{L}\left(f_{1} \cdots f_{\epsilon_{n(r)}}\left(C^{*}\right)\right)=\mathcal{L}\left(C^{*}\right) \prod_{i=1}^{n(r)} \lambda_{\epsilon_{i}}^{d} \geq \frac{r^{d} \mathcal{L}\left(C^{*}\right) \lambda_{\epsilon_{n(r)}}^{d}}{(2 \operatorname{Diam}(\Omega))^{d}} \geq \frac{\mathcal{L}\left(C^{*}\right) \min _{1 \leq j \leq n}\left\{\lambda_{j}^{d}\right\}}{(2 \operatorname{Diam}(\Omega))^{d}} \mathcal{L}(C(z, r)) .
$$

Taking $C_{0}=f_{\epsilon_{1}} \cdots f_{\epsilon_{n(r)}}\left(C^{*}\right)$ we see that our value for $\delta$ applies. Applying Lemma 3.1 yields our result.

\section{ApPliCATIONS to $\lambda$-EXPANSIONS With DELETED Digits}

Instead of considering $\lambda$-expansions where $\lambda \in\left(\frac{1}{2}, 1\right)$ and our sequences are elements of $\{0,1\}^{\mathbb{N}}$, we can consider the more general case where $\lambda \in(0,1)$ and the elements of our sequences are elements of $\mathcal{A}=\left\{a_{1}, \ldots, a_{n}\right\}$. Here $a_{j} \in \mathbb{R}$ for all $1 \leq j \leq n$, and without loss of generality we may assume that $a_{1}<\cdots<a_{n}$. We refer to $\mathcal{A}$ as our alphabet. Given $x \in\left[\frac{a_{1} \lambda}{1-\lambda}, \frac{a_{n} \lambda}{1-\lambda}\right]$ we say that a sequence $\left(\epsilon_{i}\right)_{i=1}^{\infty} \in \mathcal{A}^{\mathbb{N}}$ is a $\lambda$-expansion for $x$ with respect to $\mathcal{A}$ if

$$
x=\sum_{i=1}^{\infty} \epsilon_{i} \lambda^{i} .
$$

Expansions of this type were studied in $[4,16]$. We define the analogue of a universal expansion with respect to $\mathcal{A}$ in the natural way. Pedicini in [19] showed that every $x \in\left[\frac{a_{1} \lambda}{1-\lambda}, \frac{a_{n} \lambda}{1-\lambda}\right]$ has a $\lambda$-expansion with respect to $\mathcal{A}$ if and only if

$$
\max _{1 \leq j \leq n-1}\left(a_{j+1}-a_{j}\right) \leq \frac{\lambda\left(a_{m}-a_{1}\right)}{1-\lambda} .
$$

To the alphabet $\mathcal{A}$ we associate the set of maps $\left\{f_{j}\right\}_{j=1}^{n}$ where $f_{j}(x)=\lambda x+\lambda a_{j}$. It is straightforward to show that $\left(\epsilon_{i}\right)_{i=1}^{\infty} \in\{1, \ldots, n\}^{\mathbb{N}}$ is a coding for $x$ if and only if $\left(a_{\epsilon_{i}}\right)_{i=1}^{\infty} \in \mathcal{A}^{\mathbb{N}}$ is a $\lambda$-expansion of $x$ with respect to the alphabet $\mathcal{A}$. Therefore the attractor $\Lambda$ of $\left\{f_{j}\right\}_{j=1}^{n}$ coincides with the set of points that have a $\lambda$-expansion with respect to this alphabet. As such, when the Pedicini condition is satisfied $\Lambda=\left[\frac{a_{1} \lambda}{1-\lambda}, \frac{a_{n} \lambda}{1-\lambda}\right]$. In which case Theorem 1.4 applies and we have the following result.

Theorem 4.1. Let $\mathcal{A}=\left\{a_{1}, \ldots, a_{n}\right\}$ and suppose that $\lambda \in(0,1)$ is such that the Pedicini condition is satisfied. Then almost every $x \in\left[\frac{a_{1} \lambda}{1-\lambda}, \frac{a_{n} \lambda}{1-\lambda}\right]$ has a universal expansion with respect to $\mathcal{A}$. 
It was previously shown in [23] that when the Pedicini condition is satisfied and there exists $j$ for which $\left(a_{j+1}-a_{j}\right)<\frac{\lambda\left(a_{m}-a_{1}\right)}{1-\lambda}$, then almost every $x \in\left[\frac{a_{1} \lambda}{1-\lambda}, \frac{a_{n} \lambda}{1-\lambda}\right]$ has a continuum of expansions.

We now show that our results also translate over to cases of $\lambda$-expansions where the Pedicini condition is not satisfied. We now fix our alphabet to be $\mathcal{A}=\{0,1,3\}$. Let

$$
I_{\lambda, \mathcal{A}}:=\left\{x: x=\sum_{i=1}^{\infty} \epsilon_{i} \lambda^{i} \text { for some }\left(\epsilon_{i}\right)_{i=1}^{\infty} \in \mathcal{A}^{\mathbb{N}}\right\}
$$

The study of $\lambda$-expansions with respect to this alphabet and the set $I_{\lambda, \mathcal{A}}$ has received a lot of attention. We refer the reader to $[14,20]$ and the references therein. In [26] it was shown that for almost every $\lambda \in\left(\frac{1}{3}, \frac{4}{5}\right)$ the Lebesgue measure of $I_{\lambda, \mathcal{A}}$ is positive. Applying Theorems 1.2 and 1.4 we have the following result.

Theorem 4.2. For almost every $\lambda \in\left(\frac{1}{3}, \frac{4}{5}\right)$ almost every $x \in I_{\lambda, \mathcal{A}}$ has a continuum of $\lambda$ expansions and a universal expansion.

We remark that for all $\lambda \in\left(\frac{1}{3}, \frac{4}{5}\right)$ the Pedicini condition is not satisfied. The above theorem therefore demonstrates cases where the Pedicini condition is not satisfied yet almost every $x \in \Lambda$ has a continuum of $\lambda$-expansions and a universal expansion.

\section{OPEN PROBLEMS}

We conclude by posing some open questions and giving some general discussion.

- Let $\lambda \in\left(\frac{1}{2}, 1\right)$ and

$$
X(\lambda):=\left\{\sum_{i=0}^{n} \epsilon_{i} \lambda^{-i}: \epsilon_{i} \in\{0,1\} \text { and } n=0,1, \ldots\right\} .
$$

$X(\lambda)$ is a discrete set and may therefore be written as $\left\{y_{k}(\lambda)\right\}_{k=1}^{\infty}$ where $y_{1}(\lambda)<y_{2}(\lambda)<$ .... We introduce the following limits

$$
l(\lambda)=\liminf _{k \rightarrow \infty} y_{k+1}(\lambda)-y_{k}(\lambda) \text { and } L(\lambda)=\limsup _{k \rightarrow \infty} y_{k+1}(\lambda)-y_{k}(\lambda) .
$$

The set $X(\lambda)$ and the limits $l(\lambda)$ and $L(\lambda)$ have received a lot of attention. For more information on this topic we refer the reader to [6], [7], [1] and the references therein. The classification of those $\lambda$ for which $l(\lambda)=0$ was completed in a recent paper by Feng, see [10]. It was shown that $l(\lambda)=0$ if and only if $\lambda^{-1}$ is not a Pisot number. However, we are interested in a result stated in [7] which states that every $x \in\left(0, \frac{\lambda}{1-\lambda}\right)$ has a universal expansion with respect to the alphabet $\{0,1\}$ if $L(\lambda)=0$. Given this connection between the set $X(\lambda)$ and the existence of universal expansions the following question seems natural: For a general $\Lambda$ can we construct a set which is in some sense natural, and plays a similar role as $X(\lambda)$ does for $\lambda$-expansions? That is, does there exist $E \subset \mathbb{R}^{d}$ for which some sort of clustering property occuring within $E$ as we get further away from the origin implies the existence of universal codings for every point in $\operatorname{int}(\Omega) \cap \Lambda$. The author expects that such a set $E$ will exist. Our main motivation for 
posing this question is that we anticipate once we know how to define $E$ lots of other interesting question will arise. For example, once the analogues of $l(\lambda)$ and $L(\lambda)$ are established, when do they equal zero?

- As stated in the introduction we can construct a self-similar set with positive Lebesgue measure when $\sum_{j=1}^{n} \lambda_{j}^{d}=1$. However, the example we gave was somewhat unsatisfactory. When $p_{1}=0, p_{2}=1$ and $\lambda=\frac{1}{2}$ the images of $f_{1}([0,1])$ and $f_{2}([0,1])$ intersect in a trivial way. We would be very interested to know whether there exists an example of a self-similar set with positive Lebesgue measure when $\sum_{j=1}^{n} \lambda_{j}^{d}=1$ for which the overlaps are nontrivial. More specifically, does there exist a self-similar set with positive Lebesgue measure when $\sum_{j=1}^{n} \lambda_{j}^{d}=1$ for which there exists $1 \leq k<l \leq n$ such that $f_{k}(\Omega) \cap f_{l}(\Omega)$ has nonempty interior?

- In the case of $\lambda$-expansions with respect to the alphabet $\{0,1\}$ what can be said about the Hausdorff dimension of the set of $x \in I_{\lambda}$ with no universal expansion? For $\lambda$ sufficiently close to one it can be shown that $L(\lambda)=0$ and the set of points that do not have a universal expansion are precisely the endpoints of $I_{\lambda}$. However, we can assert that the Hausdorff dimension is positive when $\lambda \in\left(\frac{1}{2}, \lambda^{*}\right)$, where $\lambda^{*}$ is the Komornik Loreti constant. This is a straightforward consequence of the fact that $x \in I_{\lambda}$ with a unique $\lambda$ expansion cannot be universal, combined with the aforementioned results of [11] which state that for $\lambda \in\left(\frac{1}{2}, \lambda^{*}\right)$ the Hausdorff dimension of the set of $x \in I_{\lambda}$ with unique $\lambda$-expansion is positive. In particular, we would be interested in determining for which values of $\lambda \in\left(\frac{1}{2}, 1\right)$ is the Hausdorff dimension of the set of points with no universal expansion positive.

Acknowledgements The author would like to thank Tom Kempton and Nikita Sidorov for useful discussions. This work was supported by the Dutch Organisation for Scientic Research (NWO) grant number 613.001.022.

\section{REFERENCES}

[1] S. Akiyama and V. Komornik, Discrete Spectra and Pisot numbers, J. Number Theory 133 (2013), no. 2, 375-390.

[2] D. Broomhead, J. Montaldi, N. Sidorov, Golden Gaskets: Variations on the Sierpiński Sieve, Nonlinearity 17 (2004), no. 4, 1455-1480.

[3] K. Dajani, M. de Vries, Invariant densities for random $\beta$-expansions, J. Eur. Math. Soc. (JEMS) 9 (2007), no. 1, 157-176.

[4] K. Dajani, C. Kalle, Random $\beta$-expansions with deleted digits, Discrete Contin. Dyn. Syst. 18(1): 199-217, 2007

[5] Z. Daróczy, I. Katai, Univoque sequences, Publ. Math. Debrecen 42 (1993), 397-407.

[6] P. Erdős, I. Joó, V. Komornik, Characterization of the unique expansions $1=\sum_{i=1}^{\infty} q^{-n_{i}}$ and related problems, Bull. Soc. Math. Fr. 118 (1990), 377-390.

[7] P. Erdős and V. Komornik, Developments in non-integer bases, Acta Math. Hungar. 79 (1998), no. 1-2, 57-83.

[8] K. Falconer, Mathematical foundations and applications, John Wiley \& Sons, Ltd., Chichester, 2014. xxx+368 pp. ISBN: 978-1-119-94239-9 28-01.

[9] K. Falconer, The Hausdorff dimension of some fractals and attractors of overlapping construction, J. Statist. Phys. 47 (1987), no. 1-2, 123-132.

[10] D. J. Feng, On the topology of polynomials with bounded integer coefficients, arXiv:1109.1407 [math.NT]. 
[11] P. Glendinning, N. Sidorov, Unique representations of real numbers in non-integer bases, Math. Res. Letters 8 (2001), 535-543.

[12] J. Hutchinson, Fractals and self-similarity, Indiana Univ. Math. J. 30 (1981), no. 5, 713-747.

[13] T. Jordan, M. Pollicott, Properties of measures supported on fat Sierpinski carpets, Ergodic Theory Dynam. Systems 26 (2006), no. 3, 739-754.

[14] M. Keane, M. Smorodinsky, B. Solomyak, On the morphology of $\gamma$-expansions with deleted digits, Trans. Amer. Math. Soc. 347 (1995), no. 3, 955-966.

[15] J. Kigami, Analysis on fractals, Cambridge Tracts in Mathematics, 143. Cambridge University Press, Cambridge, 2001. viii+226 pp. ISBN: 0-521-79321-1.

[16] V. Komornik, A. C. Lai, M. Pedicini, Generalized golden ratios of ternary alphabets, J. Eur. Math. Soc. (JEMS) 13 (2011), no. 4, 1113-1146.

[17] V. Komornik and P. Loreti, Unique developments in non-integer bases, Amer. Math. Monthly 105 (1998), no. 7, 636-639.

[18] W. Parry, On the $\beta$-expansions of real numbers, Acta Math. Acad. Sci. Hung. 11 (1960) 401-416.

[19] M. Pedicini, Greedy expansions and sets with deleted digits, Theoret. Comput. Sci. 332 (2005), no. 1-3, 313-336.

[20] M. Pollicott, K. Simon The Hausdorff dimension of $\lambda$-expansions with deleted digits, Trans. Amer. Math. Soc. 347 (1995), no. 3, 967-983.

[21] A. Rényi, Representations for real numbers and their ergodic properties, Acta Math. Acad. Sci. Hung. 8 (1957) 477-493.

[22] N. Sidorov, Almost every number has a continuum of $\beta$-expansions, Amer. Math. Monthly 110 (2003), no. 9 , $838-842$.

[23] N. Sidorov, Combinatorics of linear iterated function systems with overlaps, Nonlinearity 20 (2007), no. 5, 1299-1312.

[24] N. Sidorov, Universal $\beta$-expansions, Period. Math. Hungar. 47 (2003), 221-231.

[25] K. Simon, B. Solomyak, On the dimension of self-similar sets, Fractals 10 (2002), no. 1, 59-65.

[26] B. Solomyak, Notes on Bernoulli convolution, Proc. Symp. in Pure Math. 72.1 (2004), 207-230, American Mathematical Society.

Utrecht University, P.O Box 80125, 3508 TC Utrecht, The Netherlands. E-Mail: SimonBAKER412@GMAIL.COM 